\title{
Baicalein modulates the radiosensitivity of cervical cancer cells in vitro via miR-183 and the JAK2/STAT3 signaling pathway
}

\author{
Hongwei Lei ${ }^{1, A, D, F}$, Jingbin Shi ${ }^{1, B, D}$, Yun Teng ${ }^{1, B, E}$, Chenghui Song ${ }^{2, C, E}$, Lijuan Zou ${ }^{1, E}$, Fuxiu Ye ${ }^{1, E}$, Haichen Zhang ${ }^{1, E}$ \\ ${ }^{1}$ Department of Radiation Oncology, The Second Hospital of Dalian Medical University, China \\ ${ }^{2}$ Maternal and Child Health Hospital of Shahekou District, Dalian, China \\ A - research concept and design; $\mathrm{B}$ - collection and/or assembly of data; $\mathrm{C}$ - data analysis and interpretation; \\ $D$ - writing the article; $E$ - critical revision of the article; $F$ - final approval of the article
}

\section{Address for correspondence \\ Haichen Zhang}

E-mail: qvvgllhnxczk@163.com

\section{Funding sources}

Dalian Science and Technology Projects 2015

(grant No. 2015E12SF150).

Conflict of interest

None declared

Received on 0 ctober 30,2020

Reviewed on November 17, 2020

Accepted on April 6, 2021

Published online on June 11, 2021

Cite as

Lei $\mathrm{H}$, Shi J, Teng $\mathrm{Y}$, et al. Baicalein modulates the radiosensitivity of cervical cancer cells in vitro via miR-183 and the JAK2/STAT3 signaling pathway. Adv Clin Exp Med. 2021;30(7):727-736. doi:10.17219/acem/135478

DOI

10.17219/acem/135478

\section{Copyright}

Copyright by Author(s)

This is an article distributed under the terms of the

Creative Commons Attribution 3.0 Unported (CC BY 3.0)

(https://creativecommons.org/licenses/by/3.0/)

\begin{abstract}
Background. Increasing radiosensitivity of cancer cells can enhance the efficacy of cervical cancer treatment.

Objectives. This study evaluated the potential roles and mechanism of baicalein in regulating the radiosensitivity of cervical cancer cells in vitro.
\end{abstract}

Materials and methods. Real-time quantitative polymerase chain reaction (RT-qPCR) was used to assess miR-183 expression in End1/E6E7 cells, Hela cells and Hela cells irradiated with X-ray (0 Gy, $1 \mathrm{~Gy}, 3 \mathrm{~Gy}$, 5 Gy, and 10 Gy). Cell Counting Kit-8 (CCK-8) method measured cell viability of Hela cells after miR-183 regulation, baicalein or R08191 treatment. Apoptosis rates were detected using flow cytometry. Thereafter, expression of $\mathrm{BCl}-2$, Bax and caspase-3 RNA was also detected through RT-qPCR. Protein concentrations of E-cadherin, N-cadherin, Vimentin in epithelial-mesenchymal transition (EMT), phospho-JAK2/STAT3, and total Janus kinase 2/signal transducer and activator of transcription 3 STAT3 (JAK2/STAT3) were examined using enzyme-linked immunosorbent assay (ELISA) methods. R08191, a JAK2/STAT3 activator, was used to activate the JAK2/STAT3 signaling pathway.

Results. The miR-183 expression was significantly lower in Hela cells compared to End1/E6E7 cells. Following upregulation of miR-183 in Hela cells, cell viability was inhibited while apoptosis was promoted. Moreover, EMT was inhibited after miR-183 over-expression. X-ray treatment markedly reduced the cell survival rate and increased miR-183 RNA expression. Baicalein treatment severely reduced the cell viability of 10-GyX-rayirradiated Hela cells, partially reversing the effect of miR-183, and also increased apoptosis and prevented EMT in irradiated cells. Y1007/8 in JAK2 and tyrosine (Tyr) residue 705 of STAT3 were phosphorylated, resulting in high expression of JAK2/STAT3, which was decreased by irradiation and baicalein treatment. R08191 activated JAK2/STAT3 signaling, promoted cell viability and EMT, and inhibited cell apoptosis, while baicalein partly reversed the functions of R08191.

Conclusions. Baicalein inhibited cell viability and EMT, and induced cell apoptosis of Hela cells, through upregulating miR-183 via inactivation of the JAK2/STAT3 signaling pathway.

Key words: irradiation, JAK2/STAT3, baicalein, miR-183 


\section{Background}

Cervical cancer is the $2^{\text {nd }}$ most common female malignant cancer worldwide. It has the $2^{\text {nd }}$ highest fatality rate in gynecologic oncology in developing countries, ${ }^{1}$ where it represents one of the most challenging public health problems. ${ }^{2}$ Recently, studies in cervical cancer have indicated a close correlation with human papillomavirus (HPV) infection, although there are still subgroups of cervical cancer patients reporting no HPV infections, suggesting that genetic factors also participate in cervical cancer progression. ${ }^{3}$ To date, the main therapeutic methods of cervical cancer are surgery, radiotherapy and chemotherapy. For patients with advanced-stage cervical cancer, radiotherapy remains the standard treatment method. ${ }^{4}$ Radiotherapy can affect the stability of DNA structure and repair through ionizing radiation (IR). ${ }^{5,6}$ If DNA damage caused by IR cannot be repaired by the DNA repair system, genomic instability, apoptosis and even death can arise in tumor cells. ${ }^{7,8}$ Based on previous studies, high doses of irradiation to the pelvic lymph nodes can increase the risk of toxicity in genitourinary (GU) and gastrointestinal (GI) cells, suggesting a need to increase the radiosensitivity of cervical cancer cells. ${ }^{9,10} \mathrm{New}$ biomarkers and targets related to radiosensitivity regulation are therefore required to obtain further information relating to cervical cancer cells.

MicroRNAs (miRNAs) are small endogenous noncoding RNAs about 22 nucleotides in length, which interact with mRNAs to negatively modulate expression, resulting in inhibition of mRNA transcription and degradation. ${ }^{11}$ In recent research, miRNAs appear to play essential roles in tumor formation and progression, ${ }^{12}$ with miRNA levels correlating with patient survival and cancer treatment. Abnormal expression of miRNAs contributes to the biological progression of cancers. ${ }^{13}$ The miR-183 is a newly detected miRNA in cervical cancer, which has already been linked to many other cancers.

The $m i R-183$ is a member of a miRNA family including $m i R-183, m i R-182$ and $m i R-96$, which is a $2-4 \mathrm{~kb}$ cluster at locus $7 \mathrm{q} 32$. The miRNAs in this cluster are abnormally expressed in hepatocellular tumors, ${ }^{14}$ colorectal cancer ${ }^{15}$ and breast cancer, ${ }^{16}$ amongst other conditions. In osteosarcoma, ectopic expression of $m i R-183$ can inhibit the migration and invasion abilities of F5M2 cells by suppressing the expression of ezrin. ${ }^{17}$ In cervical cancer, miR-183 is sequestered by $C R N D E$, acting as a sponge, resulting in upregulated expression of $C C N B 1$, leading to increased cell proliferation, migration and invasion, and reduced cell apoptosis. ${ }^{18}$ It acts as a cervical tumor suppressor, inhibiting cervical cancer cell metastasis and invasion by targeting matrix metalloproteinase-9 (MMP-9). ${ }^{19}$ However, whether $m i R-183$ could mediate radiosensitivity in cervical cancer cells is unknown. Hence, in our study, we chose an in vitro cervical cancer cell model to determine the role of $m i R-183$ in regulating radiosensitivity.
Baicalein is an active compound of the root of Scutellaria baicalensis, a traditional Chinese herbal medicine, which has activity considered to be anti-tumor, anti-viral and anti-bacterial. ${ }^{20}$ According to a previous study, baicalein can induce cell apoptosis by upregulating death receptor 5 (DR5) in colon cancer. ${ }^{21}$ Moreover, baicalein inhibits cell proliferation in MCF-7 cells and reduces HIF stability, which could also cause radiosensitization in MCF-7 cells, resulting in a high level of cell apoptosis. ${ }^{22}$ In 1 prior study of cervical cancer, baicalein induced cell apoptosis and repressed cell proliferation in an in vitro model by downregulating the Notch1/Hes1 signaling pathway. ${ }^{23}$ Baicalein has also been reported to suppress proliferation and promote apoptosis of osteosarcoma cells through upregulation of $m i R-183 .{ }^{24}$ However, we were not aware of any studies demonstrating baicalein mediating radiosensitivity in cervical cancer.

\section{Objectives}

This study evaluated the potential roles and mechanism of baicalein in regulating the radiosensitivity of cervical cancer cells in vitro. We decided to analyze the effects of baicalein on regulating the radiosensitivity of cervical cancer cells and any correlation with miR-183.

\section{Materials and methods}

\section{Cell culture}

End1/E6E7 is an epithelial HPV-16 E6/E7 transformed cell line extracted from a 43-year-old Caucasian female endometriosis patient, while the Hela cell line was the first epithelial cell line, extracted from a 31-year-old Black cervical cancer patient. Both are adherent. We used Dulbecco's modified Eagle's medium (DMEM; Gibco, Waltham, USA) supplemented with $10 \%$ fetal bovine serum (FBS), $100 \mathrm{U} / \mathrm{mL}$ of penicillin and $100 \mathrm{mg} / \mathrm{mL}$ of streptomycin, incubating the cells at $37^{\circ} \mathrm{C}$ and $5 \% \mathrm{CO}_{2}$. After incubation, Hela cells were treated with baicalein (Sigma-Aldrich, St. Louis, USA; $0 \mu \mathrm{M}, 10 \mu \mathrm{M}$ and $100 \mu \mathrm{M}$ ). Irradiated Hela cells treated with baicalein $(100 \mu \mathrm{M})$ were incubated with RO8191 (10 $\mu \mathrm{M}$; MedChemExpress (MCE), Monmouth Junction, USA), a Janus kinase 2/signal transducer and activator of transcription 3 STAT3 (JAK2/STAT3) inhibitor, for $24 \mathrm{~h}$. According to studies by Eriksson et al., $10 \mathrm{~Gy}$ $\mathrm{X}$-ray provides a significant effect compared to doses lower than $10 \mathrm{~Gy}$, and this dose has been reported to cause retarded growth of tumors. ${ }^{25}$ Normal Hela cells were irradiated with X-ray (0 Gy, 1 Gy, 5 Gy, and 10 Gy) for $3 \mathrm{~h}$. Hela cells treated by baicalein and RO8191 were irradiated with 10 Gy X-ray. After the cells received treatment and irradiation, they were used in preparations for the following experiments. 


\section{Cell transfection}

To confirm the activity of miR-183 in the Hela cell line, an inhibitor and mimics of miR-183 were compounded by GenePharma (Shanghai, China). The sequence of the miR-183 inhibitor was UAUGGCACUGGUAGAAUUCACU. Before transfection, cells were first assigned to the negative control (NC) inhibitor group, miR-183 inhibitor group, NC mimics group, or miR-138 mimics group. Hela cells were seeded on a six-well plate at a density of $1 \times 10^{5}$ cells per well. For inhibition, transfection was conducted after the confluence reached $50 \%$, while the confluence was $85 \%$ in overexpression. Thereafter, we followed the manufacturer's instructions. The NC inhibitor, miR-183 inhibitor, NC mimics, and miR-183 mimics were transfected into Hela cells using Lipofectamine 3000 (Invitrogen, Carlsbad, USA). Cells were then incubated for $24 \mathrm{~h}$. The miR-183 transfection efficiency was assessed using real-time quantitative polymerase chain reaction (RT-qPCR). After transfection, cells were collected and used for further assays.

\section{RT-qPCR}

RNA expression of miR-183 and factors related to apoptosis in the Hela and End1/E6E7 cell lines were measured using RT-qPCR. In accordance with the manufacturer's instructions for Trizol reagent (Invitrogen), total RNA was extracted from cells and reverse transcription of $10 \mu \mathrm{g}$ of total RNA was processed using a BeyoRT ${ }^{\mathrm{TM}}$ II First Strand cDNA Synthesis Kit (Beyotime, Shanghai, China). The PCR was performed using the QuantStudio ${ }^{\mathrm{TM}} 7$ Pro Real-Time PCR System (Applied Biosystems, Foster City, USA), using the following cycle conditions: pre-denaturation at $95^{\circ} \mathrm{C}$ for $5 \mathrm{~min}$, followed by 40 cycles of denaturation at $95^{\circ} \mathrm{C}$ for $30 \mathrm{~s}$, annealing at $60^{\circ} \mathrm{C}$ for $30 \mathrm{~s}$, and extension at $72^{\circ} \mathrm{C}$ for $30 \mathrm{~s}$. RNA expression levels were calculated using $2^{-\Delta \Delta \mathrm{Ct}}$ methods and GAPDH and U6 were employed to be the internal controls. The experiment was run in triplicate. The sequences of primers used are listed in Table 1.

Table 1. Sequences of primers used in RT-qPCR

\begin{tabular}{|c|c|c|}
\hline RNA & Sequences of primers & Reference \\
\hline miR-183 & $\begin{array}{l}\text { Forward, 5'- CGCGGTATGGCACTGGTAGA-3'; } \\
\text { Reverse, 5'- AGTGCAGGGTCCGAGGTATTC-3' }\end{array}$ & [26] \\
\hline $\mathrm{BCl}-2$ & $\begin{array}{l}\text { Forward, 5'- TCCATGTCTTTGGACAACCA-3'; } \\
\text { Reverse, 5'- CTCCACCAGTGTTCCCATCT-3' }\end{array}$ & {$[27]$} \\
\hline Bax & $\begin{array}{l}\text { Forward, 5'- ATGGACGGGTCCGGGGAG-3'; } \\
\text { Reverse, 5'- ATCCAGCCCAACAGCCGC-3' }\end{array}$ & [28] \\
\hline Caspase-3 & $\begin{array}{l}\text { Forward, 5'- ATGGTTTGAGCCTGAGCAGA-3'; } \\
\text { Reverse, 5'- GGCAGCATCATCCACACATAC-3' }\end{array}$ & [29] \\
\hline GAPDH & $\begin{array}{l}\text { Forward, 5'- CAAGATCATCAGCAATGCCTCC-3'; } \\
\text { Reverse, 5'- GCCATCACGCCACAGTTTCC-3' }\end{array}$ & {$[30]$} \\
\hline U6 & $\begin{array}{l}\text { Forward, 5'- CTCGCTTCGGCAGCACATATAC-3'; } \\
\text { Reverse, 5'- GGAACGCTTCACGAATTTGC-3' }\end{array}$ & {$[30]$} \\
\hline
\end{tabular}

\section{CCK-8}

To analyze the effect of baicalein on the cell survival rate of Hela cells after irradiation, a Cell Counting Kit-8 (CCK-8) assay was performed to measure cell viability and toxicity. Hela cells were seeded onto a 96-well plate at a density of $5 \times 10^{3}$ cells per well. The Hela cells were then incubated with baicalein $(0 \mu \mathrm{M}, 10 \mu \mathrm{M}$ and $100 \mu \mathrm{M})$ for $24 \mathrm{~h}, 48 \mathrm{~h}$ and $72 \mathrm{~h}$. Normal cells were irradiated with $\mathrm{X}$ - rays (0 Gy, $1 \mathrm{~Gy}, 5 \mathrm{~Gy}$, and $10 \mathrm{~Gy}$ ) for $3 \mathrm{~h}$. Baicaleintreated Hela cells were irradiated with 10 Gy X-ray. After irradiation or treatment, cells were cultured with $10 \mu \mathrm{L}$ of CCK-8 (Beyotime) for $1 \mathrm{~h}$. For cell toxicity detection, the cell survival rate was checked after irradiation using a Multiskan ${ }^{\text {TM }}$ FC Microplate Reader (Thermo Fisher Scientific, Waltham, USA) at $450 \mathrm{~nm}$ wavelength, and cell viability was measured using the same reader at the same wavelength. This experiment was repeated 3 times.

\section{Flow cytometry}

To measure the apoptosis rate of irradiated Hela cells after baicalein treatment, flow cytometry was performed using an Annexin V-FITC Apoptosis Detection Kit (Beyotime). Irradiated cells having received baicalein and RO8191 treatment were resuspended in phosphatebuffered saline (PBS). Following the manufacturers' instructions, $1 \times 10^{5}$ cells were collected and resuspended in $195 \mu \mathrm{L}$ of Annexin V-FITC binding buffer. Next, $5 \mu \mathrm{L}$ of Annevin V-FITC $(50 \mu \mathrm{g} / \mathrm{mL})$ and $10 \mu \mathrm{L}$ of propidium iodide (PI; $20 \mu \mathrm{g} / \mathrm{mL}$ ) were applied to cells and the solutions incubated for $15 \mathrm{~min}$ at room temperature without light. Following incubation, the apoptosis rate was determined using an Attune Flow Cytometer (Invitrogen). Results were collected from 3 independent experiments.

\section{ELISA}

To measure protein expression during epithelial-mesenchymal-transition (EMT) and the JAK2/STAT3 signaling pathway, the Human E-Cadherin ELISA Kit (ab233611; Abcam, Cambridge, UK), Human N-Cadherin ELISA Kit (ab254512; Abcam), Human Vimentin ELISA Kit (ab246526; Abcam), JAK2 (Phospho) [pY1007/pY1008] Human ELISA Kit, JAK2 (Total) Human ELISA Kit (Life Technologies, Carlsbad, USA), and STAT3 (Total/Phospho) Human InstantOne ${ }^{\mathrm{TM}}$ ELISA Kit (Invitrogen) were applied to measure protein densities. Enzyme-linked immunosorbent assay (ELISA) protocols strictly followed the manufacturers' instructions for each ELISA kit. The experiment was run in triplicate.

\section{Statistical analysis}

All data were presented as mean \pm standard deviation (SD) and analyzed using IBM SPSS v. 19.0 (IBM Corp., Armonk, USA) and GraphPad Prism v. 7 (GraphPad Software, 
San Diego, USA). Student's t-test (2 groups) and one-way analysis of variance (ANOVA) (3 or more groups, S-N-K method) were used to compare groups. The Bonferroni correction was used to correct significance values for multiple comparisons and $\mathrm{p}<0.05$ was considered to have statistical significance.

\section{Results}

\section{miR-183 upregulation could inhibit cell viability and promote apoptosis}

To confirm the RNA expression of miR-183 in cervical cancer cells, RT-qPCR was applied, indicating that expression of miR-183 RNA was significantly lower in Hela cells than in End/E6E7 cells (Fig. 1A). Thereafter, we used miR183 overexpression to measure its functions. Following upregulation of $m i R-183$, miR-183 RNA expression was significantly increased in Hela cells compared to the NC mimic group (Fig. 1B). The cell viability of Hela cells after miR-183 overexpression was analyzed, indicating that viability of Hela cells in the miR-183 mimic group was much lower than in the NC mimic group (Fig. 1C). The cell apoptosis rate, as detected using flow cytometry, revealed that miR-183 mimics remarkably increased the apoptosis rate of Hela cells compared with the result in the NC mimics group (Fig. 1D).

Factors related to apoptosis were also analyzed, indicating that Bcl-2 RNA expression was significantly reduced after $m i R-183$ upregulation, while Bax and caspase-3 RNA levels both greatly increased with miR-183 overexpression (Fig. 1E). We also measured the EMT of Hela cells, revealing that E-cadherin protein density was much higher in Hela cells transfected by miR-183 mimics, while N-cadherin and Vimentin levels had notably decreased (Fig. 1F).

\section{Baicalein enhanced cell viability and EMT and repressed cell apoptosis of Hela cells after irradiation}

The effects of irradiation on Hela cells were examined, indicating that the cell survival rate of Hela cells decreased in a dose-dependent manner (Fig. 2A). The viability of Hela cells irradiated with 10 Gy X-ray was measured after baicalein treatment, showing that cell viability gradually decreased as baicalein concentration increased (Fig. 2B). Flow cytometry was used to analyze cell apoptosis, indicating
A

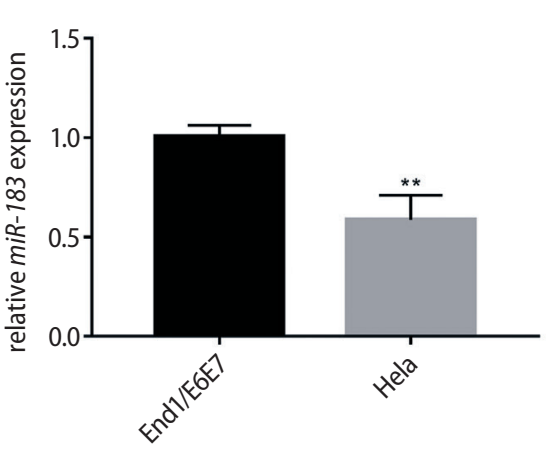

D

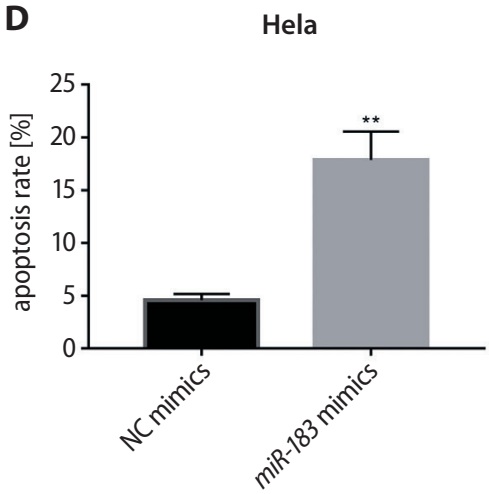

B

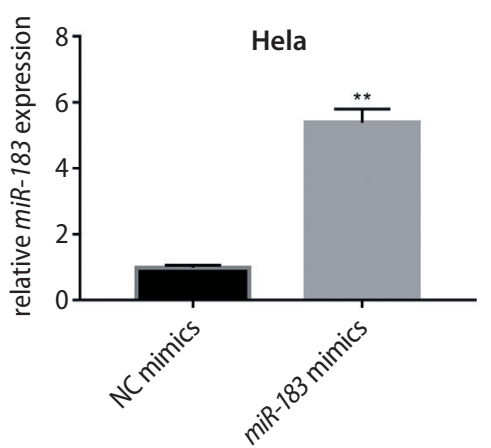

E

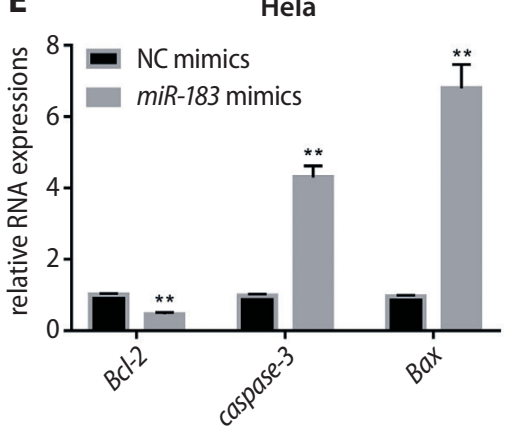

C

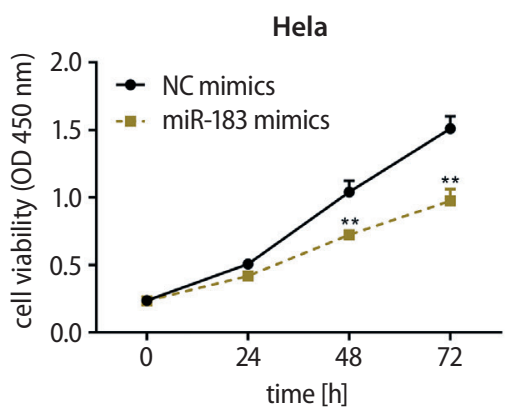

F Hela

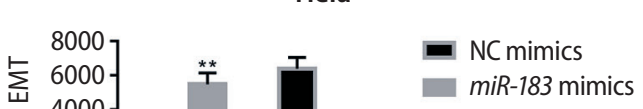

Fig. 1. Upregulation of miR-183 in Hela cells promoted cell apoptosis and inhibited cell viability and EMT

A. RNA expression of miR-183 in END1/E6E7 cells and Hela cells, as detected using RT-qPCR ( $<0.05)$; ** denotes a significant distinction from End1/E6E7 cells; B. miR-183 RNA expression was examined with RT-qPCR in Hela cells following overexpressed transfection ( $p<0.05) ; * *$ denotes a significant difference in comparison with the NC mimics group; C. Hela cell viability after miR-183 overexpression was evaluated using CCK-8 ( $<<0.05)$; ${ }^{* *}$ denotes significance in comparison with the NC mimics group; D. Flow cytometry was applied to measure Hela cell apoptosis after miR-183 overexpression ( $p<0.05)$; ${ }^{* *}$ denotes significant difference from the NC mimics group; E. BCl-2, Bax and caspase-3 RNA expression in Hela cells with miR-183 upregulation, assessed using RT-qPCR $(p<0.05) ;{ }^{* *}$ denotes significant difference from NC mimics group; F. E-cadherin, N-cadherin and vimentin protein densities, as measured using ELISA in Hela cells following miR-183 overexpression $(p<0.05){ }^{* *}$ denotes a significant difference from the NC mimics group. 
A

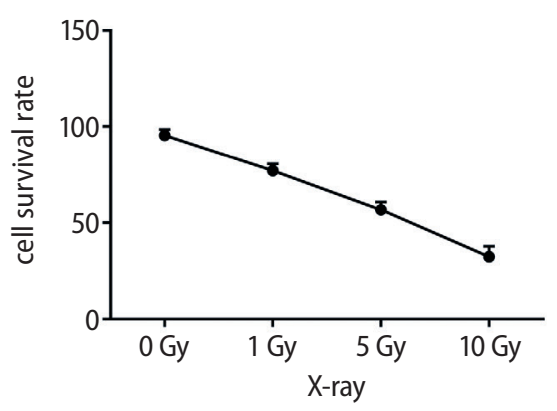

B

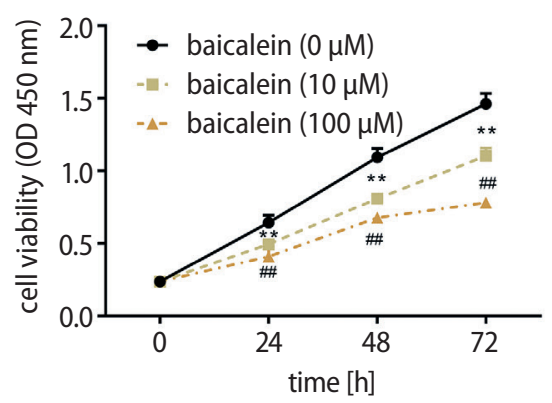

C

Hela (10 Gy)

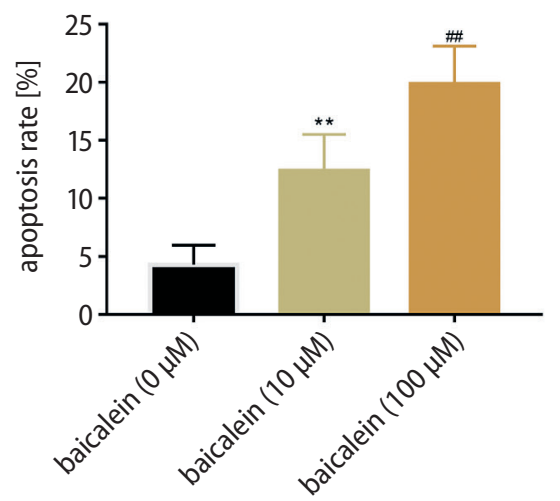

D

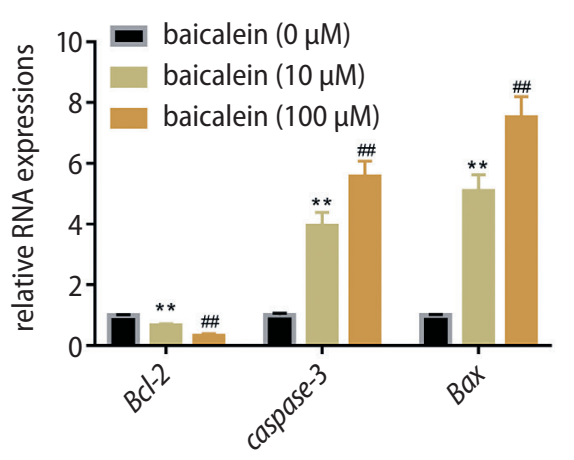

E

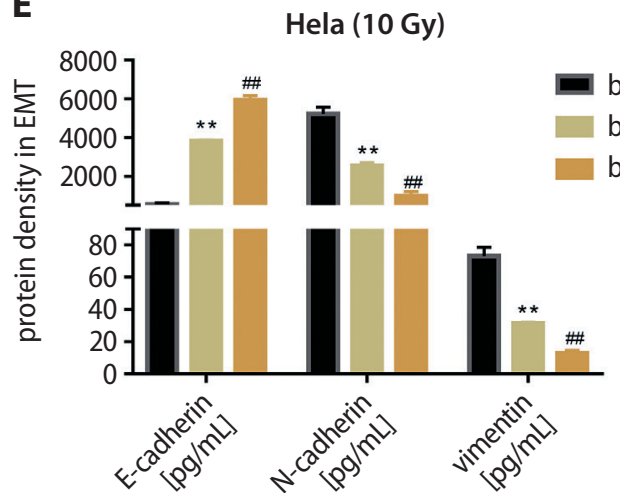

Fig. 2. Baicalein promoted cell apoptosis and inhibited cell viability and proliferation of Hela cells after irradiation

A. Cell survival rates of Hela cells treated with X-rays (0 Gy, 1 Gy, 5 Gy, and 10 Gy), evaluated using CCK-8; B. CCK-8 validation of cell viability of 10 Gy-irradiated Hela cells following baicalein treatment $(0 \mu \mathrm{M}, 10 \mu \mathrm{M}$ and $100 \mu \mathrm{M} ; \mathrm{p}<0.01)$; ** denotes a prominent difference from the $0 \mu \mathrm{M}$ group; \#\# denotes a prominent difference between the $10 \mu \mathrm{M}$ and $100 \mu \mathrm{M}$ group; C. Apoptosis rates of irradiated Hela cells analyzed using flow cytometry following treatment with baicalein $(0 \mu \mathrm{M}, 10 \mu \mathrm{M}$ and $100 \mu \mathrm{M} ; \mathrm{p}<0.01) ;{ }^{* *}$ denotes a prominent difference from the $0 \mu \mathrm{M}$ group; $\#$ denotes a prominent difference between $10 \mu \mathrm{M}$ and $100 \mu \mathrm{M}$ group; D. BCl-2, Bax and caspase-3 RNA expression was measured using RT-qPCR in Hela cells after baicalein treatment (0 $\mu \mathrm{M}$, $10 \mu \mathrm{M}$ and $100 \mu \mathrm{M}$; $\mathrm{p}<0.01) ;{ }^{* *}$ denotes a prominent difference from the $0 \mu \mathrm{M}$ group; \#\# denotes a prominent difference between the $10 \mu \mathrm{M}$ and $100 \mu \mathrm{M}$ group; E. E-cadherin, $\mathrm{N}$-cadherin and vimentin protein concentrations of irradiated Hela cells were examined using ELISA after baicalein treatment $(0 \mu \mathrm{M}$, $10 \mu \mathrm{M}$ and $100 \mu \mathrm{M}$; $\mathrm{p}<0.01) ;{ }^{* *}$ denotes a prominent difference from the $0 \mu \mathrm{M}$ group; ${ }^{\# \#}$ denotes a prominent difference between the $10 \mu \mathrm{M}$ and $100 \mu \mathrm{M}$ group.

that the apoptosis rate of irradiated Hela cells significantly increased as the concentration of baicalein increased (Fig. 2C).

The RT-qPCR was applied to measure the RNA expression of factors after baicalein treatment, showing that Bcl-2 RNA expression notably decreased, and caspase-3 and Bax RNA expression greatly increased, in a dose-dependent manner (Fig. 2D). Meanwhile, E-cadherin protein density was markedly increased, while $\mathrm{N}$-cadherin and Vimentin protein concentrations were largely reduced, again dosedependently (Fig. 2E).

\section{Baicalein promoted apoptosis and inhibited cell viability and EMT of irradiated Hela cells by upregulating miR-183}

Having confirmed the effects of miR-183 and baicalein treatment, we detected changes of miR-183 RNA expression after irradiation. The results of RT-qPCR showed that the level of miR-183 RNA was steeply increased as the density of X-ray increased (Fig. 3A). Furthermore, we examined miR-183 with baicalein treatment in Hela cells irradiated with 10 Gy X-ray, which revealed the miR-183 inhibitor largely decreased its RNA expression compared with the NC inhibitor. Baicalein treatment significantly increased miR-183 RNA level after inhibition (Fig. 3B).

We examined the cell viability of irradiated Hela cells after knockdown of $m i R-183$. In knockdown Hela cells, the miR-183 inhibitor greatly increased cell viability in comparison with the NC inhibitor group, while baicalein treatment partly reversed the promotion of cell viability caused by miR-183 inhibition (Fig. 3C). In contrast, knockdown of $m i R-183$ significantly decreased cell apoptosis compared to levels in the NC inhibitor group. In the NC inhibitor group, apoptosis activity could be restored by baicalein (Fig. 3D). To further explore cell apoptosis, related factors were also examined. The miR-183 inhibitor clearly increased Bcl-2 RNA expression when compared to the NC inhibitor, while baicalein treatment decreased the Bcl-2 RNA level. The Bcl-2 RNA level was increased by miR-183 inhibition, while caspase-3 and Bax RNA expression levels 
A

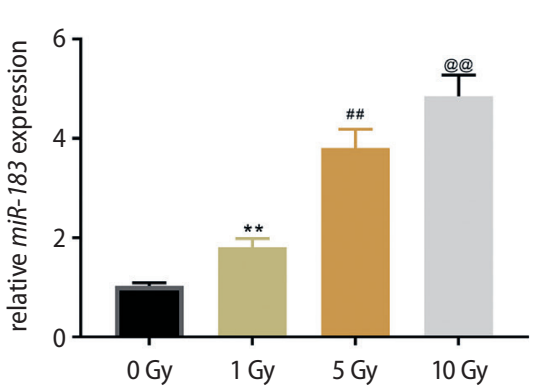

D

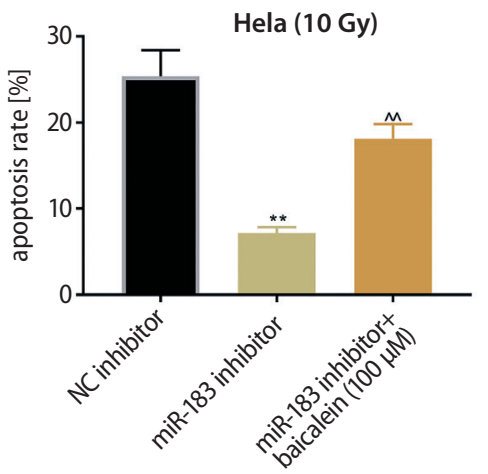

B

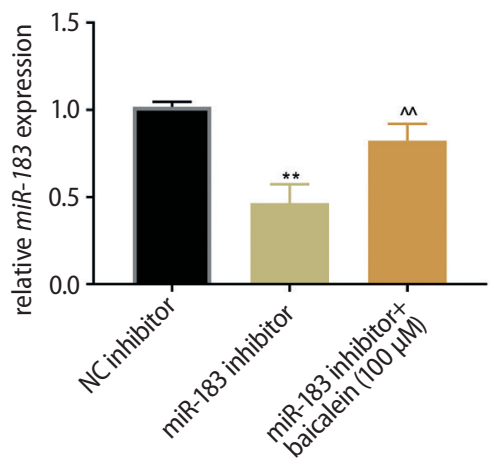

$\mathbf{E}$

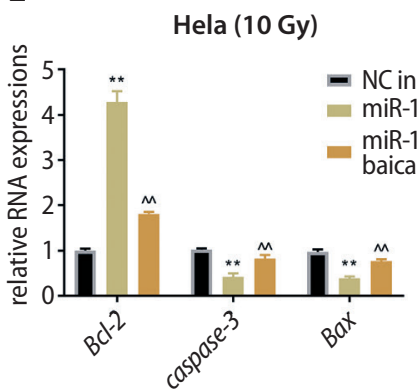

C

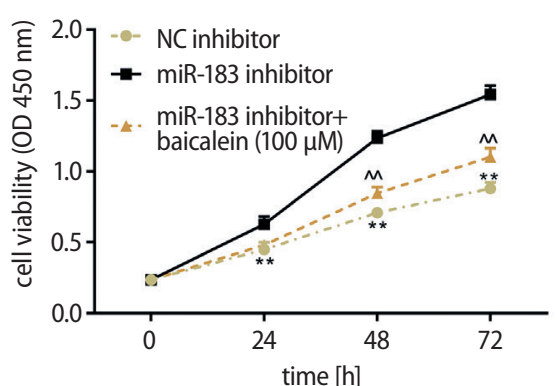

$\mathbf{F}$

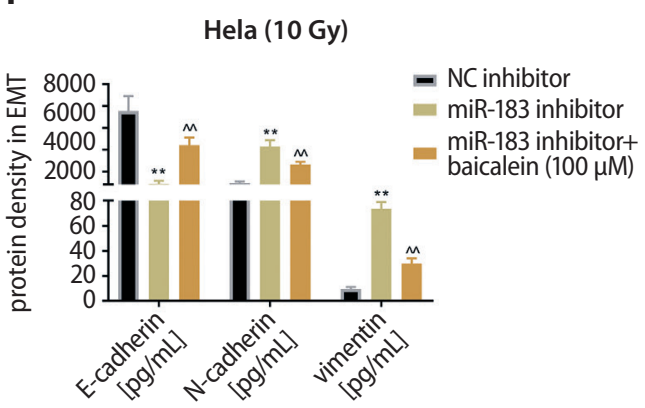

Fig. 3. Baicalein promoted apoptosis and inhibited cell viability and EMT of irradiated Hela cells by upregulating miR-183

A. miR-183 RNA expression in Hela cells after X-ray treatment (0 Gy, 1 Gy, 5 Gy, and 10 Gy) were detected using RT-qPCR ( $p<0.001)$; ** denotes a significant difference from the 0 Gy group; \#\# denotes prominent difference between the 5 Gy group and 1 Gy group; @@ denotes significant difference between the 10 Gy group and 5 Gy group; B. RT-qPCR was used to measure miR-183 RNA expression in irradiated Hela cells after miR-183 inhibition and baicalein treatment $(p<0.05) ;{ }^{* *}$ denotes notable difference from NC inhibitor group; $\wedge \wedge$ denotes significant difference between the miR-183 inhibitor group and the combined miR-183 inhibitor and baicalein $(100 \mu \mathrm{M})$ group; C. Cell viability of irradiated Hela cells after miR-183 suppression and baicalein treatment $(p<0.05) ;{ }^{* *}$ denotes notable difference from the NC inhibitor group; $\wedge \wedge$ denotes significant difference between the miR-183 inhibitor group and combined miR-183 inhibitor and baicalein $(100 \mu \mathrm{M})$ group; D. Apoptosis rates of irradiated Hela cells were evaluated using flow cytometry after downregulation of miR-183 and baicalein treatment ( $p<0.05)$; ** denotes a notable difference from the NC inhibitor group; $\wedge \wedge$ denotes significant difference between the miR-183 inhibitor group and combined miR-183 inhibitor and baicalein (100 $\mu$ M) group; E. RT-qPCR was used to detect RNA expression of Bcl-2, Bax and caspase-3 in Hela cells after irradiation, miR-183 inhibition and baicalein treatment $(p<0.05) ;{ }^{* *}$ denotes a notable difference from the NC inhibitor group; $\wedge \wedge$ denotes a significant difference between the miR-183 inhibitor group and combined miR-183 inhibitor and baicalein (100 $\mu M$ ) group; F. ELISA was applied to measure E-cadherin, $\mathrm{N}$-cadherin, and vimentin protein densities in Hela cells after irradiation, mIR-183 inhibition and baicalein treatment ( $p<0.05$ ); ** denotes a notable difference from the NC inhibitor group; $\wedge \wedge$ denotes a significant difference between the miR-183 inhibitor group and combined miR-183 inhibitor and baicalein $(100 \mu \mathrm{M})$ group.

were remarkably decreased following miR-183 downregulation. This effect was reversed after baicalein treatment (Fig. 3E).

An analysis of the EMT indicated that the miR-183 inhibitor significantly decreased E-cadherin, and increased $\mathrm{N}$-cadherin and Vimentin protein densities compared to the NC inhibitor group. Baicalein treatment attenuated the activity of miR-183 by promoting E-cadherin and inhibiting $\mathrm{N}$-cadherin and Vimentin production (Fig. 3F).

\section{Baicalein mediated apoptosis, EMT and cell viability of irradiated Hela cells via the JAK2/STAT3 signaling pathway}

The correlation between miR-183 and baicalein could be linked to the related signaling pathway. Phosphorylation of JAK2/STAT3 and total JAK2/STAT3 were both significantly higher in Hela cells than in End/E6E7 cells (Fig. 4A). Following irradiation phosphorylated JAK2/ STAT3 and total JAK2/STAT3 protein concentrations both sharply decreased with increasing doses of radiation (Fig. 4B).

We used RO8191, a JAK2/STAT3 signaling pathway activator, to examine the role of the JAK2/STAT3 pathway and to correlate this with baicalein application. The ELISA results showed that RO8191 treatment greatly increased phosphorylation of JAK2/STAT3 and total JAK2/STAT3 protein density in irradiated Hela cells, compared to untreated Hela cells after irradiation. Baicalein significantly reduced protein concentrations of JAK2/STAT3 (Fig. 4C). Moreover, the cell viability of irradiated Hela cells was measured, indicating that RO8191 usage extensively promoted cell viability of irradiated Hela cells when compared to the negative control. Conversely, baicalein usage 
A

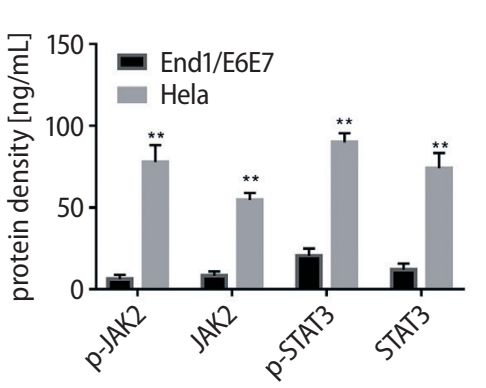

D

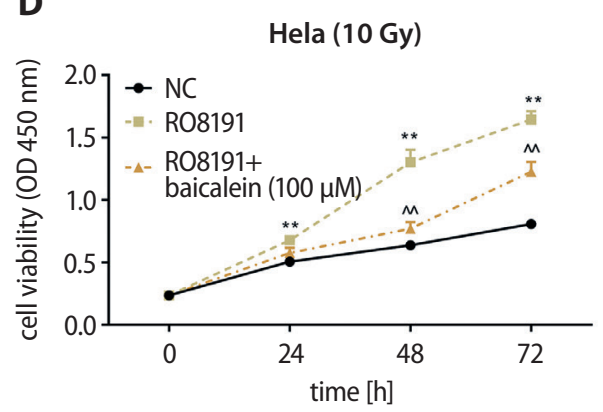

F

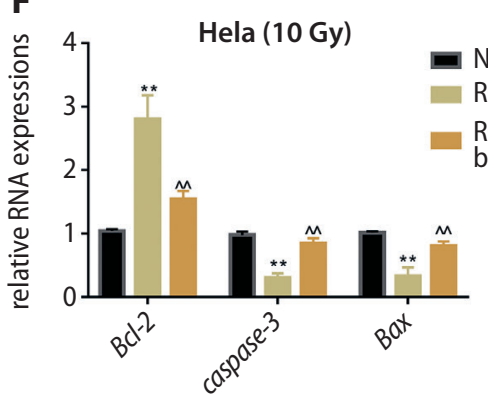

C

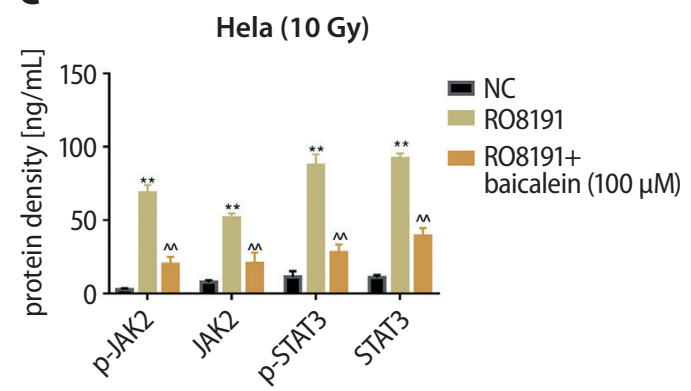

B

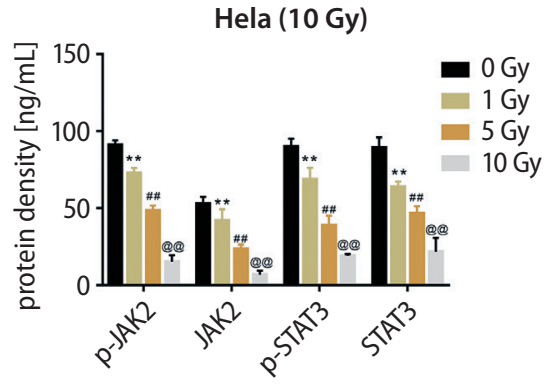

$E$

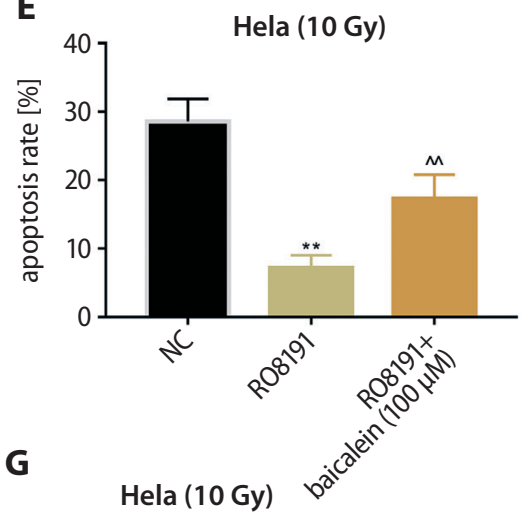

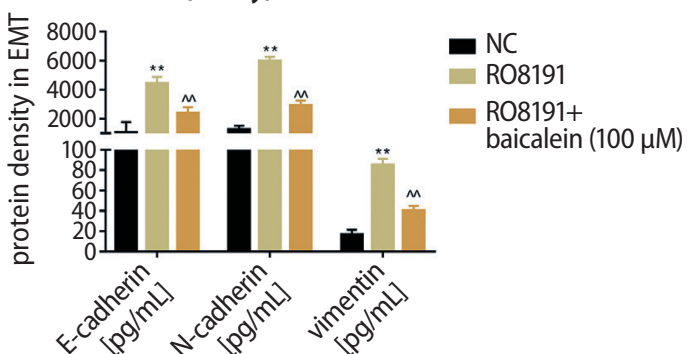

Fig. 4. Baicalein mediated irradiated Hela cell viability, cell apoptosis and EMT via the JAK2/STAT3 signaling pathway

A. Phosphorylated JAK2/STAT3 and total JAK2/STAT3 protein concentrations in End1/E6E7 cells and Hela cells $(p<0.05) ;{ }^{* *}$ denotes notable difference of protein densities from the End1/E6E7 group; B. Phosphorylation of JAK2/STAT3 and total JAK2/STAT3 protein densities in Hela cells after treatment with X-rays (0 Gy, 1 Gy, 5 Gy, and 10 Gy) were detected using ELISA ( $p<0.001$ ); ** denotes a significant difference from the 0 Gy group; \#\# signifies a notable difference between the 5 Gy group and the 1 Gy group; @@ denotes a significant difference between the 10 Gy group and the 5 Gy group; C. Protein densities of phosphorylated and total JAK2/STAT3, as detected using ELISA following RO8191 (a JAK2/STAT3 activator) usage and baicalein (100 $\mu$ M) treatment $(p<0.05) ;{ }^{* *}$ denotes a notable difference from the NC group; $\wedge \wedge$ denotes a significant difference between the RO8191 group and combined R08191 and baicalein $(100 \mu M)$ group; D. Cell viability of Hela cells after R08191 and baicalein treatment ( $<<0.05)$; ${ }^{* *}$ denotes a notable difference from the NC group; $\wedge \wedge$ denotes a significant difference between the RO8191 group and combined RO8191 and baicalein (100 $\mu M$ ) group; E. Apoptosis rates examined with flow cytometry in irradiated Hela cells following R08191 usage and baicalein treatment ( $<<0.05)$; ** denotes a notable difference from the NC group; $\wedge \wedge$ denotes a significant difference between the R08191 group and combined R08191 and baicalein (100 $\mu$ M) group; F. Bcl-2, Bax and caspase-3 RNA expression were analyzed using RT-qPCR in irradiated Hela cells after treatment with RO8191 and baicalein ( $p<0.05)$; ${ }^{* *}$ denotes a notable difference from the NC group; $\wedge \wedge$ denotes a significant difference between the RO8191 group and combined RO8191 and baicalein (100 $\mu M$ ) group; G. E-cadherin, N-cadherin, and vimentin protein densities in irradiated Hela cells after RO8191 treatment and baicalein usage were evaluated using ELISA $(p<0.05) ;{ }^{* *}$ denotes a notable difference from the NC group; $\wedge \wedge$ denotes a significant difference between the RO8191 group and combined RO8191 and baicalein $(100 \mu \mathrm{M})$ group.

reversed the promotion caused by RO8191, keeping JAK2/ STAT3 protein density and cell viability at lower levels (Fig. 4D), while increasing the cell apoptosis rate (Fig. 4E). Factors related to apoptosis were analyzed as well, showing that Bcl-2 RNA expression was significantly upregulated, while caspase-3 with Bax was significantly decreased after RO8191 treatment compared to the NC. Conversely, baicalein treatment reduced Bcl-2 RNA expression and increased caspase-3 and Bax RNA expressions (Fig. 4F).
Furthermore, the EMT was examined to measure migration and invasion abilities. These results showed that E-cadherin protein density was notably decreased, while $\mathrm{N}$-cadherin with Vimentin protein concentrations were remarkably promoted by RO8191 usage in comparison with the NC. Baicalein treatment reversed the effect of RO8191 by increasing E-cadherin protein and decreasing the $\mathrm{N}$-cadherin and Vimentin protein densities (Fig. 4G). 


\section{Discussion}

In cervical cancer treatment, chemoradiotherapy is considered an alternative solution for patients who are not good candidates for surgery, and have pelvic or para-aortic lymph node metastases. ${ }^{31}$ Unfortunately, radio-resistance of cervical cancer cells is the primary reason for failures in treatment, suggesting that increasing the radiosensitivity of cervical cancer cells could be an important method to improve the prognoses of patients. ${ }^{32}$ In this study, we examined baicalein, a tradition Chinese herbal medicine, and a newly found miRNA, miR-183, to measure their correlation and effects on cervical cancer cells.

According to prior studies, miR-183 acts as a tumor suppressor in cervical cancer cells. ${ }^{18,19}$ Therefore, we analyzed the function of miR-183 in Hela cells and its mediation of radiosensitivity in Hela cells. We first examined RNA expression, indicating that the RNA expression of miR-183 was much lower in Hela cells than in normal End1/E6E7 cells, which was similar to the findings of previous studies. We promoted expression of miR-183 to measure its role in cell outcomes. Our results showed that overexpression of miR-183 highly inhibited the viability of Hela cells and increased their cell apoptosis rate. We also analyzed factors in apoptosis to prove our result, indicating that RNA expression of Bcl-2 was significantly downregulated and Bax with caspase-3 RNA expressions was remarkably promoted.

We also examined the role of miR-183 in mediating EMT. The EMT plays important roles in the progression and metastasis of cervical cancer. ${ }^{33,34}$ With cancer cells metastasizing to other parts of the body, the prognosis for cervical cells was significantly decreased. Considering indications from former studies, we analyzed E-cadherin, $\mathrm{N}$-cadherin and Vimentin to measure miR-183 mediation of EMT in cervical cancer cells, indicating that the progression of EMT was markedly inhibited by upregulation of $m i R-183$. Therefore, we have proven that miR-183 suppressed cell viability and EMT and promoted cell apoptosis in Hela cells, suggesting that miR-183 might be a tumor suppressor of cervical cancer.

As these roles of miR-183 were confirmed, we measured the effects of irradiation and baicalein on Hela cells. After $\mathrm{X}$-ray treatment, the survival rate of Hela cells was significantly decreased in a dose-dependent manner. We selected 10 Gy X-ray-induced Hela cells for subsequent experiments, which showed that viability of Hela cells was remarkably increased dose-dependently, while the apoptosis rate was markedly increased, reflected by lowered Bcl-2 levels and increased caspase-3 and Bax. The EMT was also progressively inhibited by increased baicalein concentrations, reflected in decreased concentration of E-cadherin and increased N-cadherin and Vimentin. According to a study by Peng et al., baicalein repressed the proliferation and migration of cervical cancer cells, which also induced cervical cancer cell apoptosis and cell cycle arrest. ${ }^{35}$ In this study, baicalein was reported to play an anti-tumor role by downregulating the production of BDLNR and suppressing the PI3K/AKT signaling pathway. Baicalein also induced Hela cell apoptosis through mitochondria and death receptor pathways dose-dependently. ${ }^{35}$ In prostatic cancer, baicalein treatment increased the sensitivity of prostatic cancer cells under the mediation of 12 -LOX, ${ }^{36}$ implying that baicalein can be used as a radiosensitizer. In our study, we have detected a primary role of baicalein in mediating radiosensitivity and showed that baicalein magnified the effects of radiation on radiosensitivity regulation.

We analyzed a correlation between miR-183 and baicalein in irradiated Hela cells. This first confirmed that miR183 RNA expression was significantly upregulated by radiation in Hela cells. Thereafter, a miR-183 inhibitor was used to decrease its expression in irradiated Hela cells, which was then promoted following baicalein treatment. As seen in Fig. 3C,D, the miR-183 inhibitor promoted cell viability, but repressed apoptosis in irradiated cells, while baicalein reversed the mediations caused by knockdown of miR-183. The upregulation of BCL2 and repression of caspase-3 and Bax expression in irradiated Hela cells caused by $m i R-$ 183 downregulation was affected by baicalein, resulting in BCL2 upregulation as well as Bax and caspase-3 inhibition. Moreover, EMT was promoted by the miR-183 inhibitor, an effect which was attenuated by baicalein treatment.

We conducted a first analysis of the correlation between miR-183 and baicalein, showing that baicalein can upregulate $m i R-183$ RNA expression. Furthermore, we also detected their interactions with radiation, indicating that miR-183 and baicalein in combination could improve the efficiency of radiation treatment in Hela cells. Therefore, $m i R-183$ may be the miRNA that contributes to the radiosensitivity promoted by baicalein.

We explored a potential mechanism for baicalein increasing the radiosensitivity of Hela cells. Janus kinase 2, a member of the Janus kinase family, belongs to the nonreceptor tyrosine kinase superfamily. ${ }^{37}$ Proteins in the Janus kinase family contain 4 conserved domains: FERM, $\mathrm{SH} 2, \mathrm{JH} 2$ pseudo-kinase, and JH1 kinase. A key step in JAK kinase activation is the interaction between the $\mathrm{N}$-terminus of FERM and SH2. ${ }^{38}$ The JH1 domain contains 2 tyrosine residues (Y1007/8), which control conformation and activation through phosphorylation. ${ }^{39,40}$ Phosphorylation of Y637, Y868, Y972, and Y966 can also magnify JAK2 kinase activity, while phosphorylated Y317, Y570, Y913, and Y119 are involved in downregulation of JAK2 activation. ${ }^{41,42}$ Moreover, JAK is a potential upstream activator of STAT3, which has been shown to be activated by phosphorylation of tyrosine (Tyr) residue 705, leading to dimer formation and activation of target gene transcription. ${ }^{43-45}$ The STAT3 has been reported to be activated by oncoproteins, which are involved in oncogenesis by stimulating cell proliferation and repressing apoptosis. ${ }^{46}$ In contrast, inhibition of STAT3 results in the activation of apoptotic signaling pathways, as evidenced by upregulation of $B A X$ and CASP3 and downregulation of BCL2. ${ }^{47}$ 
In addition to proliferation regulation, the JAK2/STAT3 signaling pathway has been reported to facilitate EMT progression in oral squamous cell carcinoma, breast cancer and gastric cancer amongst other conditions. ${ }^{48-50}$ The JAK2/STAT3 signaling pathway has already been reported to be suppressed by ellagic acid, resulting in promoted cell apoptosis and inhibited cell proliferation of Hela cells. Moreover, baicalein attenuated inflammation induced by lipopolysaccharides (LPS) by suppressing JAK2 and STAT3 in RAW264.7 cells, suggesting that inhibiting the activation of the JAK/STAT signaling pathway amplifies the effects of baicalein on cells. ${ }^{51}$

We detected a correlation between the JAK2/STAT3 signaling pathway and baicalein in Hela cells. Initially, we examined the protein densities of p-JAK2, p-STAT3, t-JAK2, and t-STAT3, showing that Hela cells had higher levels of phosphorylated JAK and STAT3 and total JAK2 and STAT3, while these protein concentrations largely decreased after dose-dependently radiation. To measure the activity of JAK2/STAT3, we chose a previously demonstrated activator, RO8191 (also called CDM-3008), to activate the JAK/STAT signaling pathway. ${ }^{52}$ Based on the same study, we also used RO8191 to confirm the functions of JAK2 and STAT3, showing that RO8191 significantly increased the phosphorylation of JAK2 and STAT3, and increased the density of total JAK2 and STAT3 in Hela cells. Baicalein treatment greatly decreased the concentrations of these proteins, showing that baicalein could inhibit expression of JAK2 and STAT3 in Hela cells. Thereafter, cell viability, apoptosis and EMT were also checked, indicating that RO8191-induced high cell viability was reduced after baicalein treatment, while the inhibition of cell apoptosis caused by RO8191 was reversed. The EMT was also increased by RO8191 usage, and baicalein treatment could reduce EMT in Hela cells. This implies that baicalein might promote the radiosensitivity of Hela cells via the JAK2/ STAT3 signaling pathway.

\section{Limitations}

This study only examined the functions of baicalein in vitro. To further validate its potential in cervical cancer, animal model should be examined. This is a limitation of this study.

\section{Conclusions}

Baicalein promoted cell apoptosis and radiosensitivity, and inhibited cell viability and progression of EMT in Hela cells through miR-183 upregulation and JAK2/STAT3 inhibition, suggesting that baicalein may be a potential treatment method for increasing the radiosensitivity of Hela cells. Further studies in vivo and clinical studies are warranted to increase the knowledge about radiotherapy for cervical cancer.

\section{ORCID iDs}

Hongwei Lei (D) https://orcid.org/0000-0002-4985-9858 Jingbin Shi (D) https://orcid.org/0000-0002-1555-4044 Yun Teng (D) https://orcid.org/0000-0003-2365-1626 Chenghui Song (D) https://orcid.org/0000-0002-3119-5555 Lijuan Zou (D) https://orcid.org/0000-0002-9908-2987 Fuxiu Ye (D) https://orcid.org/0000-0001-7504-0785 Haichen Zhang (D) https://orcid.org/0000-0001-6829-3063

\section{References}

1. Suh SH, Kim JW, Aziz MF, et al. Asian society of gynecologic oncology workshop 2010. J Gynecol Oncol. 2010;21(3):137-150. doi:10.3802/ jgo.2010.21.3.137

2. Grellier N, Quéro L. Cervical cancer: Particularities in HIV patients [in French]. Bull Cancer. 2014;101(11):1040-1047. doi:10.1684/bdc.2014. 2034

3. Woodman CB, Collins SI, Young LS. The natural history of cervical HPV infection: Unresolved issues. Nature Rev Cancer. 2007;7(1):11-22. doi:10.1038/nrc2050

4. Kim JY, Byun SJ, Kim YS, Nam JH. Disease courses in patients with residual tumor following concurrent chemoradiotherapy for locally advanced cervical cancer. Gynecol Oncol. 2017;144(1):34-39. doi:10. 1016/j.ygyno.2016.10.032

5. Petrelli F, De Stefani A, Raspagliesi F, Lorusso D, Barni S. Radiotherapy with concurrent cisplatin-based doublet or weekly cisplatin for cervical cancer: A systematic review and meta-analysis. Gynecol Oncol. 2014;134(1):166-171. doi:10.1016/j.ygyno.2014.04.049

6. Alizadeh E, Orlando TM, Sanche L. Biomolecular damage induced by ionizing radiation: The direct and indirect effects of low-energy electrons on DNA. Ann Rev Phys Chem. 2015;66:379-398. doi:10.1146/ annurev-physchem-040513-103605

7. Velic D, Couturier AM, Ferreira MT, et al. DNA damage signalling and repair inhibitors: The long-sought-after Achilles' heel of cancer. Biomolecules. 2015;5(4):3204-3259. doi:10.3390/biom5043204

8. McMahon SJ, McGarry CK, Butterworth KT, et al. Cellular signalling effects in high precision radiotherapy. Phys Med Biol. 2015;60(11): 4551-4564. doi:10.1088/0031-9155/60/11/4551

9. Choi KH, Kim JY, Dong SL, et al. Clinical impact of boost irradiation to pelvic lymph node in uterine cervical cancer treated with definitive chemoradiotherapy. Medicine (Baltimore). 2018;97(16):e0517. doi:10.1097/md.0000000000010517

10. Wang W, Hou X, Yan J, et al. Outcome and toxicity of radical radiotherapy or concurrent chemoradiotherapy for elderly cervical cancer women. BMC Cancer. 2017;17(1):510. doi:10.1186/s12885-017-3503-2

11. BartelDP. MicroRNAs: Genomics, biogenesis, mechanism, and function. Cell. 2004;116(2):281-297. doi:10.1016/s0092-8674(04)00045-5

12. Papagiannakopoulos T, Kosik KS. MicroRNAs: Regulators of oncogenesis and stemness. BMCMedicine. 2008;6:15. doi:10.1186/1741-7015-6-15

13. Cortez MA, Valdecanas $D$, Zhang $X$, et al. Therapeutic delivery of miR200c enhances radiosensitivity in lung cancer. Mol Ther. 2014;22(8): 1494-1503. doi:10.1038/mt.2014.79

14. Ladeiro Y, Couchy G, Balabaud C et al. MicroRNA profiling in hepatocellular tumors is associated with clinical features and oncogene/ tumor suppressor gene mutations. Hepatology. 2008;47(6):1955-1963. doi:10.1002/hep.22256

15. Motoyama K, Inoue H, Takatsuno Y, et al. Over-and under-expressed microRNAs in human colorectal cancer. Int J Oncol. 2009;34(4): 1069-1075. doi:10.3892/ijo_00000233

16. Mattie MD, Benz CC, Bowers J, et al. Optimized high-throughput microRNA expression profiling provides novel biomarker assessment of clinical prostate and breast cancer biopsies. Mol Cancer. 2006;5:24. doi:10.1186/1476-4598-5-24

17. Zhao H, Guo M, Zhao G, et al. miR-183 inhibits the metastasis of osteosarcoma via downregulation of the expression of Ezrin in F5M2 cells. Int J Mol Med. 2012;30(5):1013-1020. doi:10.3892/ijmm.2012.1111

18. Bai X, Wang W, Zhao $P$, et al. LncRNA CRNDE acts as an oncogene in cervical cancer through sponging miR-183 to regulate CCNB1 expression. Carcinogenesis. 2020;41(1):111-121. doi:10.1093/carcin/bgz166

19. Fan D, Wang $Y$, Qui $P$, et al. MicroRNA-183 functions as the tumor suppressor via inhibiting cellular invasion and metastasis by targeting MMP-9 in cervical cancer. Gynecol Oncol. 2016;141(1):166-174. doi:10.1016/j.ygyno.2016.02.006 
20. Zhao T, Tang H, Xie L, et al. Scutellaria baicalensis Georgi. (Lamiaceae): A review of its traditional uses, botany, phytochemistry, pharmacology and toxicology. J Pharm Pharmacol. 2019;71(9):1353-1369. doi:10.1111/jphp.13129

21. Taniguchi $H$, Yoshida $T$, Horinaka $M$, et al. Baicalein overcomes tumor necrosis factor-related apoptosis-inducing ligand resistance via two different cell-specific pathways in cancer cells but not in normal cells. Cancer Res. 2008;68(21):8918-8927. doi:10.1158/0008-5472.Can-08-1120

22. Gade S, Gandhi NM. Baicalein inhibits MCF-7 cell proliferation in vitro, induces radiosensitivity, and inhibits hypoxia inducible factor. J Environ Pathol Toxicol Oncol. 2015;34(4):299-308. doi:10.1615/jenvironpatholtoxicoloncol.2015013806

23. Lian H, Hui Y, Xiaoping T, Wei T, Jiyi X, Xiaolan Y. Baicalein suppresses the proliferation of human cervical cancer cells via Notch $1 /$ Hes signaling pathway. J Cancer Res Ther. 2019;15(6):1216-1220. doi:10. 4103/0973-1482.204899

24. Zhang J, Yang W, Zhou YB, et al. Baicalein inhibits osteosarcoma cell proliferation and invasion through the miR-183/Ezrin pathway. Mol Med Rep. 2018;18(1):1104-1112. doi:10.3892/mmr.2018.9036

25. Eriksson D, Löfroth PO, Johansson L, Riklund KA, Stigbrand T. Cell cycle disturbances and mitotic catastrophes in HeLa Hep2 cells following 2.5 to $10 \mathrm{~Gy}$ of ionizing radiation. Clin Cancer Res. 2007;13 (18 Pt 2):5501s-5508s. doi:10.1158/1078-0432.Ccr-07-0980

26. Zhang Y, Wang G. MicroRNA-183 inhibits A375 human melanoma cell migration and invasion by targeting Ezrin and MMP-9. Oncol Lett. 2019;17(1):548-554. doi:10.3892/ol.2018.9603

27. Zhang Y, et al. The expression of Toll-like receptor 8 and its relationship with VEGF and Bcl-2 in cervical cancer. Int J Med Sci. 2014;11(6): 608-613. doi:10.7150/ijms.8428

28. Briton-Jones C, Lok IH, Po AL, Cheung CK, Chiu TT, Haines C. Changes in the ratio of $\mathrm{Bax}$ and $\mathrm{BCl}-2$ mRNA expression and their cellular localization throughout the ovulatory cycle in the human oviduct. J Assist Reprod Genet. 2006;23(3):149-156. doi:10.1007/s10815-0059012-2

29. Asadi M, Shanehbandi D, Asvadi Kermani T, Sanaat Z, Zafari V, Hashemzadeh S. Expression level of caspase genes in colorectal cancer. Asian Pac J Cancer Prev. 2018;19(5):1277-1280. doi:10.22034/apjcp. 2018.19.5.1277

30. Han X, Zheng J, Wang Y, Gao Z. miRNA-29a inhibits colon cancer growth by regulation of the PTEN/Akt/GSK3 $\beta$ and $W n t / \beta$-catenin signaling pathways. Oncol Lett. 2018;16(2):2638-2644. doi:10.3892/ ol.2018.8905

31. Vordermark D. Radiotherapy of cervical cancer. Oncol Res Treatment. 2016;39(9):516-520. doi:10.1159/000448902

32. Ronco G, Dillner J, Elfström KM, et al. Efficacy of HPV-based screening for prevention of invasive cervical cancer: Follow-up of four European randomised controlled trials. Lancet. 2014;383(9916):524-532. doi:10.1016/s0140-6736(13)62218-7

33. Qureshi R, Arora H, Rizvi MA. EMT in cervical cancer: Its role in tumour progression and response to therapy. Cancer Lett. 2015;356(2 Pt B): 321-331. doi:10.1016/j.canlet.2014.09.021

34. Jing L, Bo W, Yourong G, Tian W, Shixuan W, Mingfu M. Sema4C mediates EMT inducing chemotherapeutic resistance of miR-31-3p in cervical cancer cells. Sci Rep. 2019;9(1):17727. doi:10.1038/s41598-01954177-z

35. Peng Y, Gou C, Yang Y, et al. Baicalein induces apoptosis of human cervical cancer HeLa cells in vitro. Mol Med Rep. 2015;11(3):2129-2134. doi:10.3892/mmr.2014.2885
36. Lövey J, Nie D, Tóvári J, et al. Radiosensitivity of human prostate cancer cells can be modulated by inhibition of 12-lipoxygenase. Cancer Lett. 2013;335(2):495-501. doi:10.1016/j.canlet.2013.03.012

37. Liu CS, Yang-Yen HF, Suen CS, Hwang MJ, Yen JJ. Cbl-mediated K63linked ubiquitination of JAK2 enhances JAK2 phosphorylation and signal transduction. Sci Rep. 2017;7(1):4613. doi:10.1038/s41598-01704078-w

38. McNally R, Toms AV, Eck MJ. Crystal structure of the FERM-SH2 module of human Jak2. PLoS One. 2016;11(5):e0156218. doi:10.1371/journal. pone.0156218

39. VanderKuur J, Allevato G, Billestrup N, Norstedt G, Carter-Su C. Growth hormone-promoted tyrosyl phosphorylation of SHC proteins and SHC association with Grb2. J Biol Chem. 1995;270(13):7587-7593. doi:10.1074/jbc.270.13.7587

40. Zhao Y, Wagner F, Frank SJ, Kraft AS. The amino-terminal portion of the JAK2 protein kinase is necessary for binding and phosphorylation of the granulocyte-macrophage colony-stimulating factor receptor beta c chain. J Biol Chem. 1995;270(23):13814-13818. doi:10. 1074/jbc.270.23.13814

41. Argetsinger LS, Stuckey JA, Robertson SA, et al. Tyrosines 868, 966, and 972 in the kinase domain of JAK2 are autophosphorylated and required for maximal JAK2 kinase activity. Mol Endocrinol. 2010;24(5): 1062-1076. doi:10.1210/me.2009-0355

42. Robertson SA, Koleva RI, Argetsinger LS, et al. Regulation of Jak2 function by phosphorylation of Tyr317 and Tyr637 during cytokine signaling. Mol Cell Biol. 2009;29(12):3367-3378. doi:10.1128/mcb.00278-09

43. Darnell JE Jr, Kerr IM, Stark GR. Jak-STAT pathways and transcriptional activation in response to IFNs and other extracellular signaling proteins. Science. 1994;264(5164):1415-1421. doi:10.1126/science.8197455

44. Garcia R, Bowman TL, Niu G, et al. Constitutive activation of Stat3 by the Src and JAK tyrosine kinases participates in growth regulation of human breast carcinoma cells. Oncogene. 2001;20(20):2499-2513. doi:10.1038/sj.onc.1204349

45. Zhong Z, Wen Z, Darnell JE Jr. Stat3: A STAT family member activated by tyrosine phosphorylation in response to epidermal growth factor and interleukin-6. Science. 1994;264(5155):95-98. doi:10.1126/ science. 8140422

46. Bowman T, Garcia R, Turkson J, Jove R. STATs in oncogenesis. Oncogene. 2000;19(21):2474-2488. doi:10.1038/sj.onc.1203527

47. Guha P, Gardell J, Darpolor J, et al. STAT3 inhibition induces Baxdependent apoptosis in liver tumor myeloid-derived suppressor cells. Oncogene. 2019;38(4):533-548. doi:10.1038/s41388-018-0449-z

48. Chen Y, Shao Z, Jiang E, et al. CCL21/CCR7 interaction promotes EMT and enhances the stemness of OSCC via a JAK2/STAT3 signaling pathway. J Cell Physiol. 2020;235(9):5995-6009. doi:10.1002/jcp.29525

49. Kim MS, Jeong J, Seo J, Kim HS, Kim SJ, Jin W. Dysregulated JAK2 expression by TrkC promotes metastasis potential, and EMT program of metastatic breast cancer. Sci Rep. 2016;6:33899. doi:10.1038/ srep33899

50. Tao $Y$, Yang S, Wu Y, et al. MicroRNA-216a inhibits the metastasis of gastric cancer cells by targeting JAK2/STAT3-mediated EMT process. Oncotarget. 2017;8(51):88870-88881. doi:10.18632/oncotarget.21488

51. Qi Z, Yin F, Lu L, et al. Baicalein reduces lipopolysaccharide-induced inflammation via suppressing JAK/STATs activation and ROS production. Inflamm Res. 2013;62(9):845-855. doi:10.1007/s00011-013-0639-7

52. Furutani $Y$, Toguchi M, Shiozaki-Sato $Y$, et al. An interferon-like small chemical compound CDM-3008 suppresses hepatitis B virus through induction of interferon-stimulated genes. PLoS One. 2019;14(6):e02 16139. doi:10.1371/journal.pone.0216139 